\title{
Macular outer plexiform layer and outer nuclear layer thickness on spectral domain optical coherence tomography in central serous chorioretinopathy — a case of two patients
}

\author{
Maciej Czepita', Anna Machalińska², Monika Cholewa² \\ 'Department of Pathology, Pomeranian Medical University, Szczecin, Poland \\ ${ }^{2}$ Department of Ophthalmology, Pomeranian Medical University, Szczecin, Poland
}

\begin{abstract}
Increased thickness of the outer plexiform layer (OPL) of the fovea in central serous chorioretinopathy (CSC) has been previously reported. However, to our knowledge only one paper has given a concrete value of the thickness of the outer plexiform layer (OPL) of the macula in a patient with unilateral CSC. Here we report the outer plexiform and outer nuclear layer (ONL) thickness as measured with spectral domain optical coherence tomography (SD-OCT) in a patient with acute central serous chorioretinopathy in the left eye, and in a second patient with recurrent central serous chorioretinopathy in both eyes. Both patients received oral eplerenone treatment.
\end{abstract}

KEY WORDS: central serous chorioretinopathy, outer plexiform layer, optical coherence tomography

Ophthalmol J 2016; Vol. 1, No. 2, 63-66

\section{CASE HISTORY}

A 41-year-old male patient (KP) was referred to the Department of Ophthalmology of the Pomeranian Medical University in 2011. His uncorrected visual acuity (UCVA) was 0.6 in the right eye and 0.1 in the left eye. His medical history was unremarkable. Based on time domain optical coherence tomography and intravenous fluorescein angiography the patient was diagnosed with CSC in the left eye. The patient was observed for three months during which spontaneous subretinal fluid reabsorption occurred. Recurrence of his condition happened after four years. Once again he was referred to the Department of Ophthalmology of the Pomeranian Medical University. He was re-evaluated and enrolled for oral eplerenone therapy. Best corrected visual acuity (BCVA) in both his right and left eye was 1.0. SD-OCT was performed using Heidelberg Spectralis HRA + OCT in $30^{\circ}$ ART + EDI mode. A total of 25 scans of both the right and left macula were taken during both examinations. The boundaries of every layer of the macula in each scan were defined manually. Layer thickness was calculated by Heidelberg Eye Explorer software in nine subfields of the standard ETDRS grid. The values of the OPL and ONL thickness prior to treatment and at follow-up are given in Table 1 and Figure 1. During follow-up on the second day of eplerenone treatment spontaneous subretinal fluid reabsorption occurred and his OPL thickness decreased in the superior inner macula subfield (SIM), nasal inner macula subfield (NIM), inferior inner macula subfield (IIM), and temporal inferior macula subfield (TIM). The outer nuclear layer (ONL) also expe- 
Table 1. Outer plexiform layer and outer nuclear layer thickness of the left eye of patient KP during the examination before treatment and after day 2 of treatment. All values are given in $\mu \mathrm{m}$

\begin{tabular}{|l|c|c|c|c|c|c|c|c|}
\hline OPL/ONL thickness in subfields & SIM & SOM & NIM & NOM & IIM & IOM & TIM & TOM \\
\hline Examination before treatment & $48 / 64$ & $29 / 70$ & $37 / 51$ & $27 / 63$ & $42 / 43$ & $26 / 61$ & $57 / 49$ & $33 / 60$ \\
\hline Examination at day 2 & $40 / 73$ & $30 / 69$ & $33 / 62$ & $29 / 61$ & $34 / 53$ & $26 / 60$ & $40 / 70$ & $32 / 62$ \\
\hline
\end{tabular}

SIM — superior inferior macula; SOM — superior outer macula; NIM — nasal inferior macula; NOM — nasal outer macula; IIM — inferior inner macula; IOM — inferior outer macula; TIM — temporal inferior macula; TOM — temporal outer macula

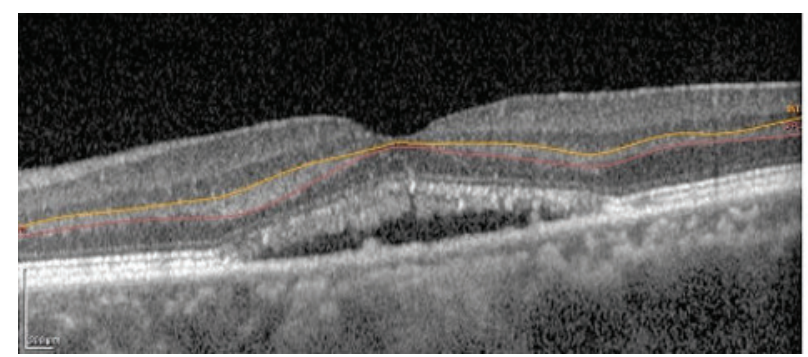

2015-09-09, 0S, Patient KP

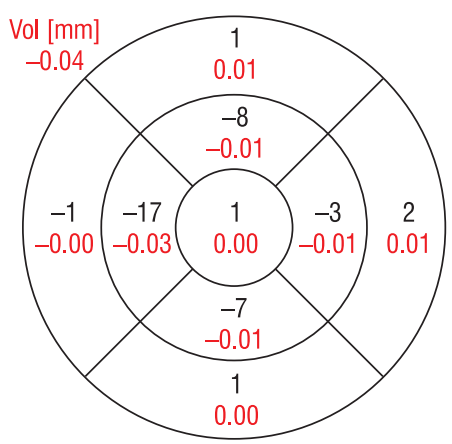

Outer plexiform layer thickness in patient KP 2015-09-22 compared to 2015-09-09

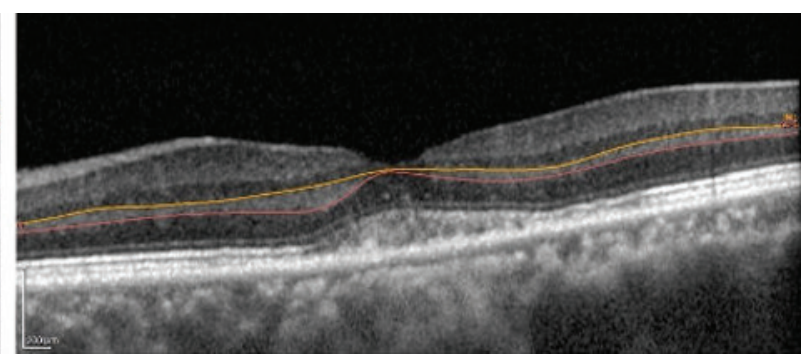

2015-09-22, OS, Patient KP

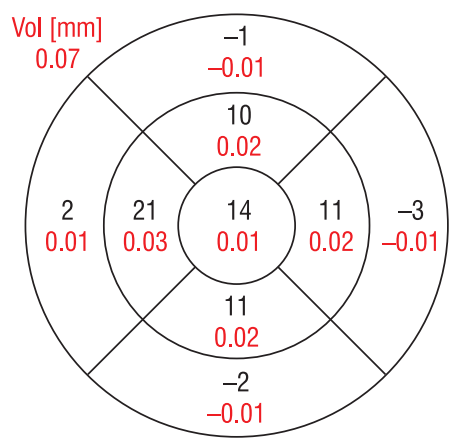

Outer nuclear layer thickness in patient KP 2015-09-22 compared to 2015-09-09

FIGURE 1. The values of the OPL and ONL thickness prior to treatment and at follow-up in patient KP

rienced changes during treatment. In all but the superior outer macula subfield (SOM), nasal outer macula subfield (NOM), and inferior outer macula subfield (IOM) the thickness decreased slightly. In the remaining subfields an evident increase in thickness occurred.

Our second patient, a 37-year-old male patient (MB), presented to the Department of Ophthalmology of the Pomeranian Medical University with subjective decreased visual acuity in the left eye lasting for three months. His medical history was unremarkable. At presentation his uncorrected visual acuity (UCVA) was 1.0 both in the right and left eye. Intravenous fluorescein angiography disclosed a serous retinal detachment in the macula of the left eye, which was proven on SD-OCT. Based on these findings the patient was diagnosed with CSC in the left eye and qualified for treatment with oral eplerenone. SD-OCT using a Heidelberg Spectralis
HRA + OCT in $30^{\circ}$ ART + EDI mode was performed on day 12 after initiation of treatment and on day 32. A total of 25 scans of the right and left macula were taken during both examinations. The boundaries of every layer of the macula in each scan were defined manually. Layer thickness was calculated by Heidelberg Eye Explorer software in nine subfields of the standard ETDRS grid. The results of both OPL and ONL during both visits are given in Table 2 and Figure 2. On day 12 after initiation of treatment the subretinal fluid was absorbed and reappeared on day 32. At this time, OPL thickness increased in all but the nasal outer macula subfield (NOM), inferior inner macula subfield (IIM), inferior outer macula subfield (IOM), and temporal outer macula subfield (TOM). ONL thickness remained stable in superior outer macula (SOM) and temporal outer macula (TOM) subfields, decreased in superior inferior macula (SIM), nasal inferior macula 
Table 2. Outer plexiform layer and outer nuclear layer thickness of the left eye of patient MB during examination on day 12 and 32. All values are given in $\mu \mathrm{m}$

\begin{tabular}{|l|c|c|c|c|c|c|c|c|}
\hline OPL/ONL thickness in subfields & SIM & SOM & NIM & NOM & IIM & IOM & TIM & TOM \\
\hline Examination at day 12 & $29 / 80$ & $25 / 72$ & $37 / 68$ & $27 / 67$ & $29 / 66$ & $27 / 58$ & $26 / 64$ & $27 / 68$ \\
\hline Examination at day 32 & $36 / 73$ & $27 / 72$ & $39 / 66$ & $25 / 69$ & $28 / 67$ & $25 / 61$ & $34 / 55$ & $26 / 68$ \\
\hline
\end{tabular}

SIM — superior inferior macula; SOM — superior outer macula; NIM — nasal inferior macula; NOM — nasal outer macula; IIM — inferior inner macula; IOM — inferior outer macula; TIM — temporal inferior macula; TOM — temporal outer macula

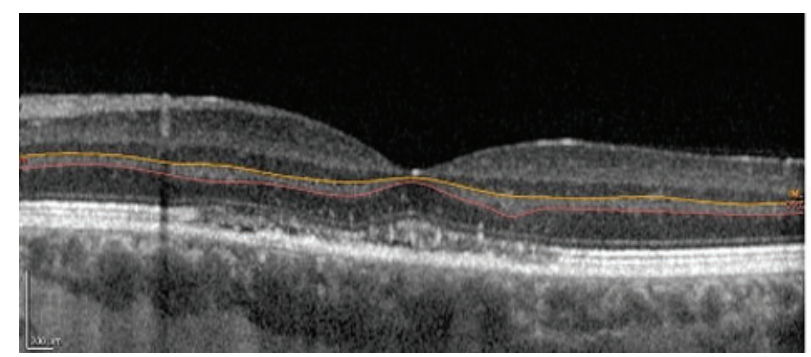

2015-08-28, 0S, Patient MB

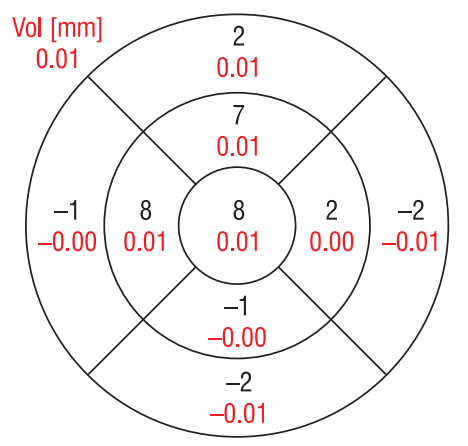

Outer plexiform layer thickness in patient $\mathrm{MB}$ 2015-09-17 compared to 2015-08-28

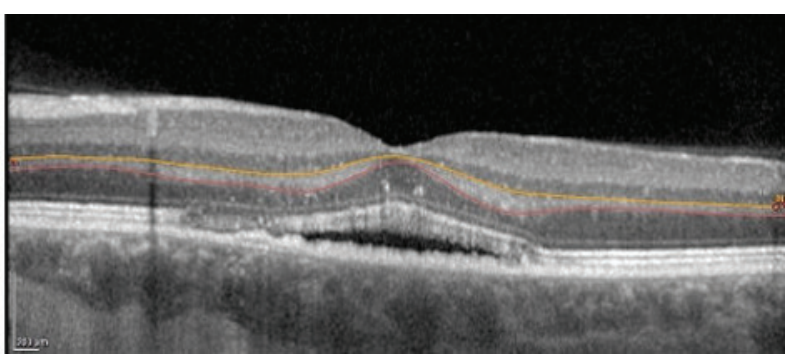

2015-09-17, 0S, Patient MB

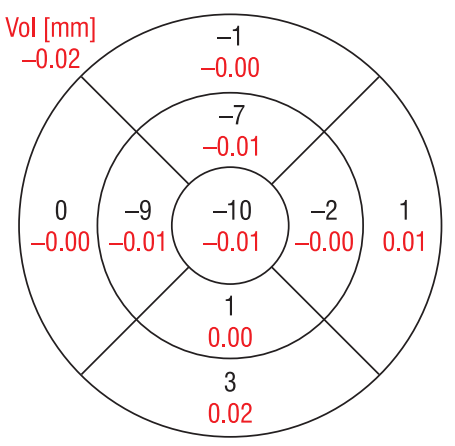

Outer nuclear layer thickness in patient MB 2015-09-17 compared to 2015-08-28

FIGURE 2. The values of the OPL and ONL thickness prior to treatment and at follow-up in patient MB

(NIM), inferior outer macula (IOM), and temporal inferior macula (TIM) subfields, and increased in nasal outer macula (NOM), inferior inner macula (IIM), and inferior outer macula (IOM) subfields.

\section{DISCUSSION}

Central serous chorioretinopathy is a common disorder involving detachment of the neurosensory retina and/ or pigmented epithelium due to subretinal fluid accumulation and choroidal vessel hiperpermeability [1]. It has been associated with a number of conditions such as collagen vascular diseases, IBD, endogenous hypercorticolism (Cushing disease) and factors such as type A personality, exogenous corticosteroid use, antihistamine use [2].

Recent studies using SD-OCT on patients with CSC have revealed different intraretinal abnormalities. Ahlers et al. demonstrated diffuse hyper-re- flectivity at the level of the outer nuclear layer and outer plexiform layer in 16 out of 18 patients with CSC, attributing this to probable accumulation of subretinal fluid within these layers [3]. Similar hyper-reflectivity on SD-OCT was observed by Ouyang et al. in a 49-year-old patient with unilateral CSC, believing it to be caused by slight tilting during scan acquisition [4]. Moreover, Plateroti et al. have described intraretinal deposits within the outer plexiform layer visible on SD-OCT in 7 out of 13 patients examined, with acute CSC appearing at different times in the course of the disease [5]. In our opinion the hyper-reflectivity of the OPL causing the observed increased outer plexiform layer thickness as detected in SD-OCT in our CSC patients represents backscatter of reflected light from the Henle fibre layer, which is disfigured by the underlying subretinal fluid [6]. We speculate that macular pigment released in CSC from the Henle 
fibre layer could also have an influence on the hyper-reflectivity of the outer plexiform layer as observed in SD-OCT by minimising the amount of scattered light; the light backscatter from the Henle fibres could then be better detectable. Macular pigment has been noted to be released from the Henle fibre layer and inner plexiform layer in patients with both acute and chronic CSC [7]. Further studies to evaluate the potential role of macular pigment of the hyper-reflectivity of the OPL are warranted. Interestingly, we also observed changes in the thickness of the outer nuclear layer above areas of subretinal fluid. The thickness of this layer decreased, while the thickness of the outer plexiform layer increased. The opposite happened in the case of reabsorption of subretinal fluid.

\section{REFERENCES}

1. Rogers A, Duker JS. Central serous retinopathy. In: Rapid diagnoses in ophthalmology: Retina. Elsevier, New York 2008: 178-181.

2. Putnam CM, Kinerk WT, Bassi CJ. Central serous chorioretinopathy produces macular pigment profile changes. Optom Vis Sci 2013; 90: e206-e212.

3. Ahlers C, Geitzenauer W, Stock G, Golbaz I, Schmidt-Erfuhrt U, Prünte C. Alterations of intraretinal layers in acute central serous chorioretinopathy. Acta Ophthalmol 2009; 87: 511-516.

4. Ouyang Y, Walsh AC, Keane PA, Heussen FM, Pappuru RK, Sadda SR. Different phenotypes of the appearance of the outer plexiform layer on optical coherence tomography. Graefes Arch Clin Exp Ophthalmol 2013; 251: 2311-2317.

5. Plateroti AM, Witmer MT, Kiss S, D'Amico DJ. Characteristics of intraretinal deposits in acute central serous chorioretinopathy. Clin Ophthalmol 2014; 8: 673-676.

6. Alkabes M, Pichi F, Lembo A, Nucci P. Pathological visualization of Henle's fiber layer using spectral domain optical coherence tomography. Invest Ophthalmol Vis Sci 2012; 53: 808.

7. Sasamoto Y, Gomi F, Sawa M, Tsujikawa M, Hamasaki T. Macular pigment optical density in central serous chorioretinopathy. Invest Ophthalmol Vis Sci 2010; 51: 5219-5125. 